\title{
ESTIMATION OF DRIVERS' BEHAVIOR CRITERIA AFFECTING ROAD SAFETY BY THE ANALYTIC HIERARCHY PROCESS APPLICATIONS
}

\author{
Danish Farooq ${ }^{1}$, Sarbast Moslem ${ }^{2}$, Rana Faisal Tufail ${ }^{3}$ \\ ${ }_{1,2}$ Department of Transport Technology and Economics, Budapest University of Technology and \\ Economics, 1111 Budapest, Müegyetem rakpart 3, Hungary \\ ${ }^{3}$ College of Architecture and Environment, Sichuan University 610065, China
}

Received 10 July 2019; accepted 15 August 2019

\begin{abstract}
Driver behavior has been found one of the most influential factors on road safety. Driver behavior identification is key to solve road safety issues related to driver behavior. This study aims to identify and quantify the significant driver behavior factors affecting road safety by using Analytic Network Process applications. The driver behavior dataset is collected from a self-reported questionnaire survey from novice and experienced drivers. The ANP pairwise comparison results are utilized to rank the driver behavior factors based on normalized weights. The ANP results showed that "driving without alcohol use" was the most significant driver behavior criteria for both groups. For experienced drivers, the lowest rank observed driver behavior criteria is "maintain safe gap between vehicles". While for novice drivers the lowest rank observed driver behavior criteria is "use personal intelligent assistant". Furthermore, the Analytic network process (ANP) model found that most of driver behavior factors are interrelated based on driver groups responses. Finally, the Kendall's rank correlation is applied to estimate the concordant degree between evaluator groups. The results evaluated that there is a medium correlation between the driver groups but not a perfect agreement. The study results can help traffic safety authorities to focus on significant driver behavior criteria to solve road issues.
\end{abstract}

Keywords: drivers' behaviour criteria, analytic network process, significance, road safety, Kendall's correlation.

\section{Introduction}

Human factors have been estimated to be a sole or leading causal factor in approximately $90 \%$ of road traffic accidents (NHTSA, 2008; Evans, 2004; Lewin, 1982; Rumar, 1985). According to Hungarian Central Statistical office data there were 625 road fatalities in 2017, a $2.9 \%$ increase when compared to 2016
(KSH, 2017). The situation analysis of the Road Safety Action Program observes that most of the accidents are caused by human factors, therefore influencing them becomes the most important target of road safety actions (OECD/ITF, 2016). The basic driver behavior factors which directly influence on road safety were such as driving behavior, driving experience and driver's perception

${ }^{1}$ Corresponding author: Farooq.danish@mail.bme.hu 
of traffic risks (De Oña et al., 2014). Mostly drivers involve in behaviors that cause safety issues to both themselves and to other road users also. Many of these driver behavior factors are dynamic, conscious rule violations, while others are the types of errors due to less driving experience, momentary mistakes, failure to perform function or inattention, the latter often related to age (Stanton and Salmon, 2009; Wierwille et al., 2002).

The Driver Behavior Questionnaire (DBQ) is one of the most widely used instruments for measuring driving style. To assess deviant driving behavior, the Driver Behavior Questionnaire (DBQ) was first developed as a tool in the related studies 1990s (Parker et al., 1995; Reason et al., 1990). The findings of the previous studies have shown that selfreported driving behaviors are associated with both active and passive traffic accidents (Bener et al., 2007; Bener and Crundall, 2008).

There are several studies which evaluated driver behavior criteria affecting road safety. However, some of these studies applied multi-criteria decision making (MCDM) methods for road safety analysis (Farooq et al., 2019; Chen et al., 2015; Kanuganti et al., 2017; Fancello et al., 2018; Haghighat, 2011). The ANP approach was proposed by Saaty as a generalization approach to dependence and feedback of the well-known Analytic Hierarchy Process (AHP). ANP provides a deeper insight into complex decisions based on PCs than simple statistical survey (Saaty, 1996; Saaty, 2005). The ANP approach can measure all possible interrelations among observed factors by utilizing the pairwise comparison (PC) survey based on Saaty scale (Duleba and Moslem, 2019). While the Kendall's rank correlation is a non- parametric statistic test used in previous studies to measure the concordance degree between rater groups (Ahmad et al., 2015; Duleba and Moslem, 2018; Couso et al., 2018).

The study was undertaken to investigate the most significant driver behavior factors affecting road safety by using Analytic Network Process (ANP) Applications. The study highlighted the significant driver behavior criteria for two evaluator groups and measured the interrelations between the observed criteria. Furthermore, Kendall's coefficient of concordance is used to estimate the concordance degree among evaluator groups. The study suggests that the most significant driver behavior factors should be focused to solve road safety issues related to driver behavior.

\section{Methodology}

\subsection{Sample Characteristics}

The study utilized the driver behavior questionnaire (DBQ) as a tool to identify and quantify risky driver behavior factors affecting road safety in Budapest city. DBQ can be a useful tool for predicting drivers' involvement in traffic accidents based on their self-reported driving behavior. The results were used to verify the correlation between driver behavior and involvement in traffic accidents (Af Wåhlberg et al., 2015).

The Driver Behavior Questionnaire (DBQ) is designed on Saaty's scale which includes thirteen driver behaviour items affecting road safety for the convenience of pairwise comparisons (PCs). The questionnaire survey involved two groups of transportation engineering experts having the driving experience listed here ((Group 
A: Experienced drivers who have more than twenty years driving experience) + (Group B: Novice drivers who have less than two years driving experience)). These drivers have sound knowledge about transport safety due to their relevant profession (Department of Transport Technology and Economics). For DBQ data collection, the participants were approached and interviewed to fill the questionnaire on perceived road safety issues in Budapest University of Technology and Economics. The evaluator number of the questionnaire survey is not evidently statistically representative. However, the ANP and the AHP methods provide a deeper insight based on the PCs than the simple statistical surveys (Duleba and Moslem, 2019). Solomon (2006) highlighted in his phenomenon "Wisdom of crowds" that 20 evaluators can provide an extreme opinion. Some important characteristics of participants related to age, gender and driving experience are mentioned below in Table 1.

\section{Table 1}

Sample Characteristics

\begin{tabular}{|l|c|c|}
\hline \multicolumn{1}{|c|}{ Variables } & Group A & Group B \\
\hline $\mathrm{N}$ & 40 & 40 \\
\hline Age & & 21.635 \\
Mean & 43.274 & 2.037 \\
$\mathrm{SD}$ & 4.672 & 0.815 \\
\hline Gender $(1=$ male,0=female) & & 0.317 \\
Mean & 0.873 & \\
SD & 0.253 & 1.852 \\
\hline Driving Experience & & 1.341 \\
Mean & 21.326 & \\
SD & 3.714 & \\
\hline
\end{tabular}

\subsection{Driver Behavior Criteria Affecting Road Safety}

Driver behavior has been found complex and uncertain to study road safety issues. Driving behavior identification was considered as the most important part of traffic studies which provides useful information generally in three main fields such as road safety analysis, microscopic traffic simulation and intelligent transportation systems (ITS) (Bifulco et al., 2014). Thirteen driver behavior factors which directly influence on road safety are considered for the study. These factors are important for safe movements of drivers themselves and for other road users also as discussed in previous studies
(De Oña et al., 2014; Farooq and Juhasz, 2018; ACEM, 2004; Pradhan et al., 2014; Bener et al., 2007; Watson, 2004). These factors are symbolized from $\mathrm{F} 1$ to $\mathrm{F} 13$ for the convenience of analysis. The driver behavior factors along with symbols were listed here. F1: Driver attention, F2: Driver visual perception, F3: Obeying speed limits, F4: Use personal intelligent assistant, F5: Respect yielding rules, F6: Maintain safe gap between vehicles, F7: Avoid frequently changing lanes, F8: Comply traffic lights, F9: Applying brakes at hazardous situations, F10: Deterrence of punish for traffic violations, F11: Traffic scan accurately, F12: Obeying overtaking rules, F13: Driving without alcohol use. 


\subsection{Analytic Network Process (ANP)}

ANP is a dynamic process that reflects the real situation of the complex problems in which factors act in a non-hierarchical way. The analytic network process (ANP) was developed by Saaty which can examine the complex interrelationships among factors and decision levels (Saaty, 1996; Saaty, 2005; Farooq and Sarbast, 2019).

In this study, the ANP approach is utilized to identify and quantify the most significant driver behavior factors affecting road safety. Super decision software is used to analyze the questionnaire data for evaluator groups such as novice drivers and experienced drivers. According to ANP approach, the first step of the analysis is to fill the pairwise comparison matrices (PCM) to set the factors level of the PCs. Saaty suggested the fundamental scale of 1-9 to compare between factors (Saaty, 1996). The fundamental ratio scale consists of numerical values which provide different sorts of information is shown in Table 2. For example, digit one represents equal importance of both factors and digit nine represents extreme importance of one factor over another.

Table 2

Judgment Scale of Relative Importance for Pairwise Comparison

\begin{tabular}{|c|c|c|}
\hline $\begin{array}{c}\text { Numerical } \\
\text { values }\end{array}$ & Verbal scale & Explanation \\
\hline 1 & Equal importance of both factors & Two factors contribute equally \\
\hline 3 & $\begin{array}{c}\text { Moderate importance of one factor over } \\
\text { another }\end{array}$ & $\begin{array}{l}\text { Experience and judgment slightly favor one } \\
\text { factor over another }\end{array}$ \\
\hline 5 & Strong importance of one factor over another & $\begin{array}{l}\text { Experience and judgment strongly favor one } \\
\text { factor over another }\end{array}$ \\
\hline 7 & $\begin{array}{c}\text { Very strong importance of one factor over } \\
\text { another }\end{array}$ & One factor is very strongly dominant \\
\hline 9 & Extreme importance of one factor over another & $\begin{array}{l}\text { One factor is favored by at least an order of } \\
\text { magnitude }\end{array}$ \\
\hline $2,4,6,8$ & Intermediate values & Used to compromise between two judgments \\
\hline
\end{tabular}

Source: (Saaty, 1996)

The consistency analysis of selected factors in super matrix was examined by applying Saaty's Consistency Index (CI). Consistency Ratio $(C R)<0.1$ (Saaty, 2005) was determined as follow Eq. (1).

$C I=\frac{\lambda_{\max }-n}{n-1}$

where $C I$ is the consistency index, $\lambda_{\text {max }}$ is the maximum eigenvalue and $n$ is the number of rows in the matrix. $C R$ can be determined by Eq. (2).
$C R=\frac{C l}{R I}$

where $R I$ is the random consistency index. If $A$ is a consistency matrix in relation with vector $W$ depicted as $A . W=\lambda_{\max } . W$ . Then eigenvector $W$ can be calculated as $\left(A-\lambda_{\max }\right) \cdot W=0$, where $\lambda_{\max }$ is the maximum eigenvalue of the matrix $\mathrm{A} . \lambda_{\text {max }}$ is also known as principal eigenvalue of the matrix A. The threshold was also determined by Saaty, the PCM can be considered as acceptable from inconsistency point of view if $\mathrm{CR}<0.1$. 
Pairwise evaluations have been aggregated in the case of multiple evaluators. For aggregating collected data, the geometric mean aggregation method was applied, because it is the most common group preference aggregation method in AHP and ANP approaches (Aczél and Saaty, 1983; Saaty, 1989).

If " $h$ " evaluators exist in the procedure, then Eq. (3):

$f\left(x_{1}, x_{2}, \ldots, x_{h}\right)=\sqrt[h]{\prod_{k=1}^{h} x_{a_{i j k}}}$

Where $a_{i j k}$ denotes the aij element of the evaluator " $k$ ".

After generating the aggregated matrices, the driving weight vector scores was further determined in the procedure. For measuring the eigenvectors of the aggregate matrices, the following method was applied as Eq. (4):

$w_{A i}=\frac{w_{j}}{w} \frac{w_{i j}}{\sum_{k=1}^{n} w_{i k}}=\left(\frac{w_{j}}{w} \frac{1}{\sum_{k=1}^{n} w_{i k}}\right) w_{i j}$

where $j=1, \ldots, m$ and $w_{j}>0$ represents the related weight coordinate from the previous level; $w_{i j}>0$ is the eigenvector computed from the matrix in the current level, $w_{A i}$ is the calculated weight score of current level's elements $(i=1, \ldots, n)$. The consistency ratio (CR) was acceptable to complete ANP analysis.

The main eigenvector of each PCM represented the synthesis of the numerical judgments established at each level of the network (Saaty, 1989).

\subsection{Kendall's Concordance Test}

Kendall's coefficient of concordance (W), was proposed by Kendall and Smith (1939). W is a normalization of the statistic of the Friedman test, which is considered as a non-parametric statistic technique and can be used for a set of criteria to highlight the agreement level among different raters (Gibbons and Kendall, 1990; McLeod, 2005; Abdi, 2007). To estimate the agreement degree or concordant degree between group A and group B, the Kendall's coefficient of concordance is measured using Eq. (5).

$R_{i}=\sum_{j=1}^{m} r_{i j}$

Where $R_{i}$ is the aggregated ranking of the factor $i, r_{i j}$ is the rank given to factor $i$ by the evaluator group $j$, $m$ is the number of rater groups rating the $n$ factors.

$R=\frac{m(n+1)}{2}$

$\mathrm{R}$ (Eq. 6) is the mean of the Ri values.

$S=\sum_{i=1}^{n}\left(R_{i}-R\right)^{2}$,

Where $S$ is a sum of squares statistic (Eq. 7) deviations over the row sums of ranking $R_{i}$.

Following that, Kendall's "W" statistic can be obtained from the following formula Eq. (8):

$W=\frac{12 s}{m^{2}\left(n^{2}-n\right)}$

After applying the formula, the result will be a number that determines the agreement degree among the different groups. The Kendall's agreement degree value occurs between zero and one (Duleba and Moslem, 2018; Couso et al., 2018) as shown in Table 3. 
Table 3

Kendall's Agreement Degree (W) Scale

\begin{tabular}{|c|c|}
\hline Correlation Coefficient & Interpretation \\
\hline 1 & Perfect agreement \\
\hline $0.9-1$ & very high agreement \\
\hline $0.7-0.9$ & High agreement \\
\hline $0.4-0.7$ & Medium agreement \\
\hline $0.2-0.4$ & Low agreement \\
\hline $0-0.2$ & very low agreement \\
\hline 0 & No agreement \\
\hline
\end{tabular}

Source: (Kendall and Smith, 1939)

\section{Results and Discussion}

The study estimated the driver behavior criteria affecting road safety and measured the correlation between evaluator groups by using Analytic Network Process (ANP) applications. Firstly, ANP was utilized to quantify the significant driver behavior factors based on drivers' responses. Accordingly, pairwise comparison method was utilized to assign each criteria a quantitative weight. In the pairwise comparison method, the criteria were arranged in square matrix. Based on the measured parameter such as normalized weight, the driver behavior factors were ranked from one to thirteen for both evaluator groups as shown in Table 4 . The ANP analysis results showed that "driving without alcohol use" was the most important factor based on driver's response data for both rater groups. Also, the results can be justified according to Hungarian driving laws that there is zero tolerance policy towards drinking and driving ( $\mathrm{WHO}$, 2015). Furthermore, for experienced drivers the second most significant observed factor was "obeying overtaking rules". It was analyzed that dangerous overtaking accounted for $41 \%$ of all drivers who died in traffic in 2006 (Walker, 2007). While the lowest rank observed factor for experienced drivers was "maintain safe gap between vehicles". Moreover, for novice drivers the second most observed significant factor was "comply traffic lights". One of the possible causes for the high number of crashes and injuries is due to beating traffic lights (Subramaniam et al., 2007). While the lowest rank observed factor for novice drivers was "use personal intelligent assistant". These preferences make decisions more flexible to solve the variety of road safety problems. 
Table 4

Final Normalized Weights for Driver Behavior Factors Related to Road Safety

\begin{tabular}{|c|c|c|c|c|}
\hline \multirow{2}{*}{ Factors } & \multicolumn{2}{|c|}{ Group A } & \multicolumn{2}{c|}{ Group B } \\
\cline { 2 - 5 } & Normalized weight & Ranking & Normalized weight & 9 \\
\hline F1 & 0.059 & 8 & 0.047 & 11 \\
\hline F2 & 0.047 & 11 & 0.043 & 8 \\
\hline F3 & 0.041 & 12 & 0.053 & 13 \\
\hline F4 & 0.06 & 7 & 0.041 & 12 \\
\hline F5 & 0.066 & 6 & 0.042 & 4 \\
\hline F6 & 0.038 & 13 & 0.065 & 3 \\
\hline F7 & 0.048 & 10 & 0.08 & 6 \\
\hline F8 & 0.081 & 5 & 0.165 & 7 \\
\hline F10 & 0.05 & 9 & 0.06 & 5 \\
\hline F11 & 0.094 & 3 & 0.058 & 10 \\
\hline F12 & 0.083 & 4 & 0.063 & 1 \\
\hline F13 & 0.101 & 2 & 0.046 & 0.237 \\
\hline
\end{tabular}

Secondly, Analytic Network Process (ANP) was further utilized to describe the structure of the driver behavior model by measuring the interrelations between specified factors for evaluator groups as shown in Figures 1(a) and 1(b). These connections indicated the flow of influence between the factors based on driver responses on perceived road safety issues. The analysis results showed that most factors were interrelated for both groups and few factors were not interrelated. Figures 1(a) and 1(b) were observed slightly different from each other, where Figure 1(a) has four interrelations more than Figure 1(b) which are presented in dotted lines. In the applied survey, seventy-eight comparisons were observed between the factors. Results showed that sixty-three $(78-15=63)$ factors are interrelated in case of group (A) and fiftynine $(78-19=59)$ factors are interrelated in case of group (B). The four higher in numbers observed interrelations between observed factors for experienced drivers are listed here; (i) driver visual perception and applying brakes at hazardous situation (ii) driver visual perception and driving without alcohol use (iii) obeying the speed limit and use personal intelligent assistant (iv) use personal intelligent assistant and deterrence of punish for traffic violation.

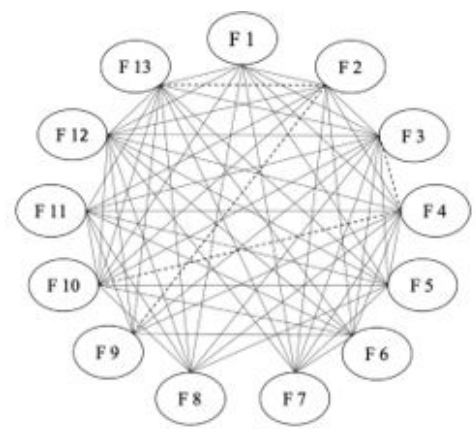

(a) Experienced drivers

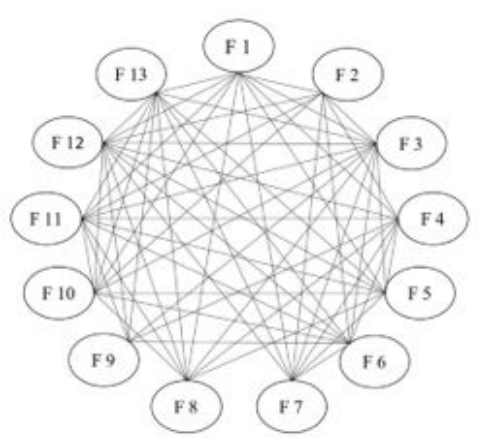

(b) Novice drivers

Fig. 1.

The Interdependent Relationships Between Driver Behavior Criteria for Evaluator Groups 
Finally, Kendall's rank correlation was applied to estimate the agreement degree or concordant degree between evaluator driver groups based on driver behavior criteria affecting road safety. Kendall's coefficient of concordance (W) was measured for assessing agreement among rater groups. Kendall's correlation value (W) ranges from 0 (no agreement) to 1 (complete agreement). According to Kendall's coefficient of concordance (W), the value observed is 0.5632 as shown in Table 5. This value evaluated that there is a medium agreement between the rater groups but not a perfect aggreement.

Table 5

ANP Results for Estimation of Kendall's Coefficient of Concordance (W)

\begin{tabular}{|c|c|c|c|c|}
\hline Factor & Rank of Group A & Rank of Group B & $R_{i}$ & $\left(R_{i}-R^{2}\right.$ \\
\hline F1 & 8 & 9 & 17 & 9 \\
\hline F2 & 11 & 11 & 22 & 64 \\
\hline F3 & 12 & 8 & 20 & 36 \\
\hline F4 & 7 & 13 & 20 & 36 \\
\hline F5 & 6 & 12 & 18 & 16 \\
\hline F6 & 13 & 4 & 17 & 9 \\
\hline F7 & 10 & 3 & 13 & 49 \\
\hline F8 & 5 & 2 & 7 & 1 \\
\hline F9 & 9 & 6 & 15 & 16 \\
\hline F10 & 3 & 7 & 9 & 25 \\
\hline F11 & 4 & 5 & 12 & 4 \\
\hline F12 & 2 & 10 & 2 & 144 \\
\hline F13 & 1 & 1 & $\mathrm{R}=14$ & $\mathrm{~W}=0.5632$ \\
\hline n13 & $\mathrm{m}=2$ & $\mathrm{~S}=410$ & & \\
\hline
\end{tabular}

\section{Conclusion}

The study was designed to use Analytic Network Process (ANP) applications for evaluation and ranking of the driver behavior's factors affecting road safety. The self-reported questionnaire survey based on Saaty's scale was used as a tool for evaluator groups evaluation by considering thirteen important driving attitudes. The pairwise comparison method was used to assign each criteria a quantitative weight in such a way to satisfy the rank quantitatively. For this purpose, the normalized weight values were calculated for each factor. The ANP results showed that "driving without alcohol use" was the most significant driver behavior factor based on driver response data for both groups. The results also evaluated that "maintain safe gap between vehicles" was the lowest observed factor for experienced drivers. While for novice drivers, the lowest rank observed factor was "use personal intelligent assistant”. These results help to understand which driver behavior factors are more significant from experienced and novice drivers' point of view. Furthermore, ANP model results found that most of driver behaviour factors are interrelated to each other based on drivers' responses by evaluator groups in DBQ. However, experienced drivers selected four extra interrelations between specified factors as compared to novice drivers. ANP results were further used to measure Kendall's rank correlation for the estimation of the agreement degree or 
concordant degree between evaluator groups. The results showed that there was a medium agreement between evaluator groups but not perfect agreement.

The overall study results can help to identify the significant driver's behavior criteria affecting road safety for experienced and novice drivers. ANP applications enable the decisionmakers to better understand the complex and uncertain individual driver behavior in the decision-making and subsequently improves the reliability of the decision. However, a large amount of driver behavior data with advance multi-criteria decision-making technique can help to evaluate the driver behaviour criteria more comprehensively.

\section{References}

Abdi, H. 2007. The Kendall rank correlation coefficient. Encyclopedia of Measurement and Statistics. Sage, Thousand Oaks, 508-510.

ACEM. 2004. In-depth investigations of accidents involving powered two wheelers. Final report number: 2, Avenue de la Joyeuse Entrée 1 - 1040. Brussels.

Aczél, J.; Saaty, T. L. 1983. Procedures for synthesizing ratio judgements, Journal of mathematical Psychology 27(1): 93-102.

Af Wåhlberg, A. E.; Barraclough, P.; Freeman, J. 2015. The Driver Behavior Questionnaire as accident predictor; A methodological re-meta-analysis, Journal of Safety Research 55: 185-212.

Ahmad, I.; Tang, D.; Wang, T.; Wang, M.; Wagan, B. 2015. Precipitation trends over time using Mann-Kendall and spearman's rho tests in swat river basin Pakistan, Advances in Meteorology Article ID 431860, 15 pages.
Bener, A.; Crundall, D.; Haigney, D.; Bensiali, A. K.; Al-Falasi, A. S. 2007. Driving behavior stress, error and violations on the road: a cross-cultural comparisons study, Advances in Transportation Studies 12: 5-14.

Bener, A.; Crundall, D. 2008. Effects of driver behavior on accident involvement: The role of gender and driver behavior in road traffic crashes, International Journal of Crashworthiness 13(3): 331-336.

Bifulco, G. N.; Galante, F.; Pariota, L.; Spena R. M.; Gais D. P. 2014. Data Collection for Traffic and Drivers' Behavior Studies: a large-scale survey, Procedia-Social and Behavioral Sciences 111: $721-730$.

Chen, F.; Wang, J.; Deng, Y. 2015. Road safety risk evaluation by means of improved entropy TOPSIS-RSR, Safety science 79: 39-54.

Couso, I.; Strauss, O.; Saulnier, H. 2018. Kendall's rank correlation on quantized data: An interval-valued approach, Fuzzy Sets and Systems 343: 50-64.

De Oña, J.; de Oña, R.; Eboli, L.; Forciniti, C.; Mazzulla, G. 2014. How to identify the key factors that affect driver perception of accident risk, A comparison between Italian and Spanish driver behaviour, Accident Analysis and Prevention 73: 225-235.

Duleba, S.; Moslem, S. 2018. Sustainable Urban Transport Development with Stakeholder Participation, an AHP-Kendall Model: A Case Study for Mersin, Sustainability 10(10): 36-47.

Duleba, S.; Moslem, S. 2019. Examining Pareto optimality in analytic hierarchy process on real Data: An application in public transport service development, Expert Systems with Applications 116: 21-30.

Evans, L. 2004. Traffic Safety. Science Serving Society, Inc., Bloomfield Hills. 
Fancello, G.; Carta, M.; Fadda, P. 2018. Road intersections ranking for road safety improvement: Comparative analysis of multi-criteria decision-making methods, Transport Policy 80: 188-196.

Farooq, D.; Juhasz, J. 2018. Analysis of Young Driver Behaviour related to Road Safety Issues in Pakistan and Hungary. In Proceedings of the $3^{\text {rd }}$ World Congress on Civil, Structural, and Environmental Engineering (CSEE'18) Budapest, Hungary, Paper No. ICTE 109.

Farooq, D.; Moslem, S.; Duleba, S. 2019. Evaluation of Driver Behavior Criteria for Evolution of Sustainable Traffic Safety, Sustainability 11(3142).

Farooq, D.; Moslem, S. 2019. Evaluation and Ranking of Driver Behavior Factors Related to Road Safety by Applying Analytic Network Process, Periodica Polytechnica Transportation Engineering (in print) doi: https://doi. org/10.3311/PPtr.13037.

Gibbons, J. D. 1990. Kendall M. Rank Correlation Methods, 5th ed.; Edward Arnold: London, UK.

Haghighat, F. 2011. Application of a multi-criteria approach to road safety evaluation in the Bushehr Province, Iran, Promet - Traffic \& Transportation 23(5): 341-352.

Kanuganti, S.; Agarwala, R.; Dutta, B.; Bhanegaonkar, P. N.; Singh, A. P.; Sarkar, A. K. 2017. Road safety analysis using multi criteria approach: A case study in India, Transportation research procedia 25: 4649-4661.

Kendall, M. G.; Smith, B. B. 1939. The problem of $\mathrm{m}$ rankings, Annals of Mathematics Statistics 10: 275-287.

KSH. 2018. Road accidents involving personal injury (2001-2017). KSH, Budapest, Hungary. Available from Internet: <http://www.ksh.hu/docs/eng/ xstadat/xstadat_annual/i_ods002.html >. [Accessed: 02/06/2019].
Lewin, I. 1982. Driver training: A perceptual-motor skill approach, Ergonomics 25(10): 917-24.

McLeod, A. I. 2005. Kendall rank correlation and MannKendall trend test. R Package Kendall.

NHTSA (National Highway Traffic Safety Administration). 2008. National Motor Vehicle Crash Causation Survey. U.S Department of Transportation: Washington, DC, USA.

OECD/ITF. 2016. Road Safety Annual Report. OECD: Paris, France; ITF: London, UK.

Parker, D.; Reason, J. T.; Manstead, A. S. R.; Stradling, S. G. 1995. Driving errors, driving violations and accident involvement, Ergonomics 38: 1036-1048.

Pradhan, A. K.; Kaigang, Li.; Bingham, R. C.; SimonsMorton, B.; Ouimet, C. M.; Shope, J. T. 2014. Peer passenger influences on male adolescent drivers' visual scanning behavior during simulated driving, Journal of Adolescent Health 54(5): S42-S49.

Reason, J.; Manstead, A.; Stradling, S.; Baxter, J.; Campbell, K. 1990. Errors and violations on the roads: a real distinction? Ergonomics 33 (10-11): 1315-1332.

Rumar, K. 1985. The Role of Perceptual and Cognitive Filters in Observed Behavior. In Human Behavior and Traffic Safety, L. Evans and R. C. Schwing, eds. New York: Plenum Press.

Saaty, T. L. 1989. Group decision making and the AHP. In The analytic hierarchy process, Springer. Berlin, Heidelberg, 59-67.

Saaty, T. L. 1995. Transport planning with multiple criteria: the analytic hierarchy process applications and progress review, Journal of Advanced Transport 29(1): 81-126. 
Saaty, T. L. 1996. Decision making with dependence and feedback: The analytic network process. Pittsburgh, PA: RWS Publications.

Saaty, T. L. 2005. Theory and Applications of the Analytic Network Process. Pittsburgh: RWS Publications.

Solomon, M. 2006. Groupthink versus the wisdom of crowds: The social epistemology of deliberation and dissent, Southern Journal of Philosophy 44: 28-42.

Stanton, N. A.; Salmon, P. M. 2009. Human error taxonomies applied to driving; generic driver error taxonomy and its implications for intelligent transport systems, Safety Science 47: 227-237.

Subramaniam, K.; Phang, W. K.; Hayati, K. S. Traffic light violation among motorists in Malaysia, IATSS Research 31: 67-73.

Walker, I. 2007. Drivers overtaking bicyclists: Objective data on the effects of riding position, helmet use, vehicle type and apparent gender, Accident Analysis and Prevention 39: 417-425.
Watson, B. 2004. How effective is deterrence theory in explaining driver behaviour: A case study of unlicensed driving?. In Proceedings of the Road Safety Research, Policing and Education Conference, Perth, WA.

Wierwille, W.W.; Hanowski, R. J.; Hankey, J. M.; Kieliszewski, C. A.; Lee, S. E.; Medina, A.; Keisler, A. S.; Dingus, T. A. 2002. Identification and evaluation of driver errors: overview and recommendations. Report No: FHWA-RD-02-003, U.S Department of Transportation, Federal Highway Administration.

WHO. 2015. Global status report on road safety. World Health Organization. Available from Internet: <https:// www.who.int/violence_injury_prevention/road safety_status/2015/en/>. [Accessed: 05 July 2019].

\section{ijtte 375}

